\title{
EXISTENCE AND UNIQUENESS OF EQUILIBRIUM STATES OF A ROTATING ELASTIC ROD
}

\author{
M.B.M. ELGINDI \\ Department of Mathematics \\ University of Wisconsin-Eau Claire \\ Eau Claire, WI 54702-4004
}

(Received April 7, 1992 and in revised form June 25, 1992)

\begin{abstract}
A flexible rod is rotated from one end. The equilibrium equation is a fourth order nonlinear two-point boundary value problem which depends on two parameters $\lambda$ and $\alpha$ representing the importance of centrifugal effects to flexural rigidity and the angle between the rotation axis and the clamped end, respectively. Previous studies on the existence and uniqueness of solution of the equilibrium equation assumed $\alpha=0$. Among the findings of these studies is the existence of a critical value $\lambda_{c}$ beyond which the uniqueness of the "trivial" solution is lost. The computations of $\lambda_{c}$ required the solution of a nonlinear bifurcation problem. On the other hand, this work is concerned with the existence and uniqueness of solution of the equilibrium equation when $\alpha \neq 0$ and in particular in the computations of a critical value $\lambda_{c}$ such that the equilibrium equation has a unique solution for each $\alpha \neq 0$ provided $\lambda<\lambda_{c}$. For small $\alpha \neq 0$ this requires the solution of a nonlinear perturbed bifurcation problem.
\end{abstract}

KEY WORDS AND PHRASES. Existence and uniqueness of equilibrium states, rotating rods, nonlinear eigenvalue problems, fourth order two-point nonlinear boundary value problems, Schauder fixed point theorem, perturbed bifurcation problems, perturbation solution.

AMS SUBJECT CLASSIFICATION CODES. 49G99, 73H05, 73K15.

\section{INTRODUCTION.}

Consider an elastic rod of uniform cross section and density which makes an angle $\alpha, 0 \leq \alpha \leq \frac{\pi}{2}$, with the horizontal. The rod is being rotated from one end that is supported by a bushing. The other end is free (Figure 1). This problem serves as a model for many important engineering problems. Among the previous studies are the papers by Odeh and Tadjbakshs [1] and Wang [2], [3]. Both [1] and [2] considered the case when $\alpha=0$. In [1] it is shown that the "trivial" solution becomes unstable as the rotational velocity exceeds a certain critical value and that the problem possesses nontrivial solutions for such rotational velocity. The proof of existence of the critical rotational velocity required the solution of a nonlinear bifurcation problem and the proof of the existence of the nontrivial solutions was done using several applied analysis techniques, some of which will be used in this work. [2] is concerned with both the analytical (using the perturbation methods) and the numerical computations of the nontrivial solutions. The case $0<\alpha \leq \frac{\pi}{2}$ is treated analytically and numerically in [3]. 
In this paper we are concerned with proof of the existence and uniqueness of solution of the equilibrium equation when $0<\alpha \leq \frac{\pi}{2}$. In particular we are interested in providing a critical value of the rotational velocity such that for each $\alpha \neq 0$ the equilibrium equation has a unique solution for rotational velocities that are less than the critical value. For small $\alpha \neq 0$ this requires the solution of a nonlinear perturbed bifurcation problem.

Our formulations of the problem go along the same lines as those of [1] and [2] for the case when $\alpha=0$. In fact, the only change is in one of the boundary conditions. We assume that the gravity effects are negligible and that the rod is thin enough so that it can be treated as an elastica. Figure 2 shows the coordinate system. A moment balance on an element of length $d s^{\prime}$ gives (Figure 3)

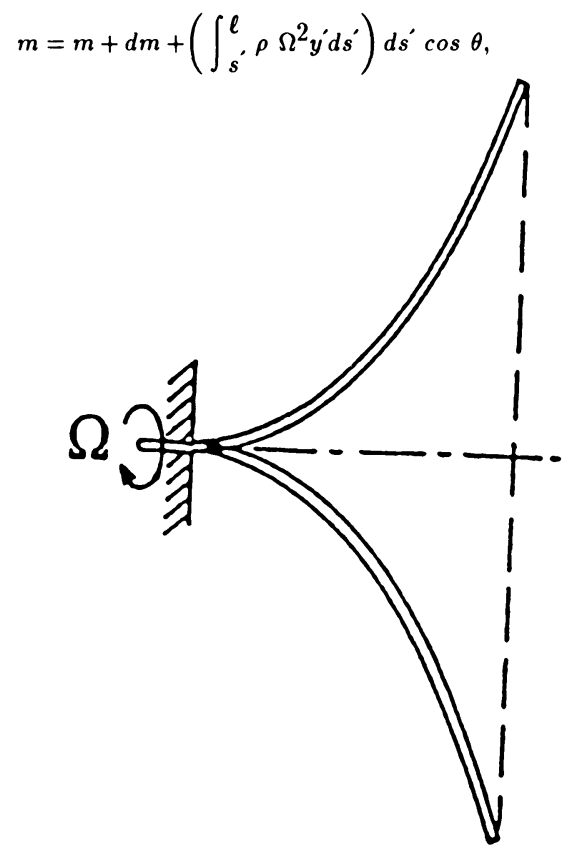

Figure 1: The axially rotating rod

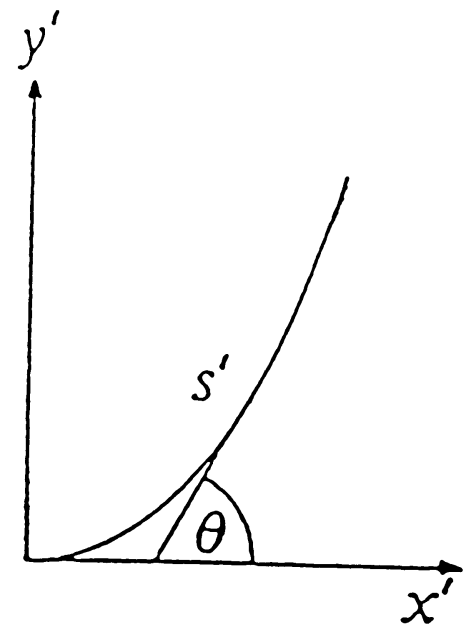

Figure 2: The coordinate system 


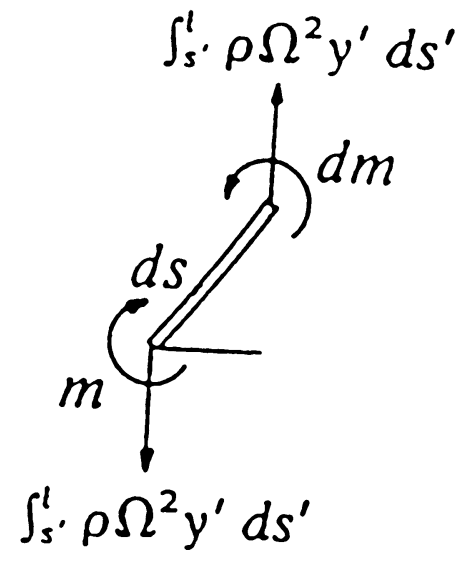

Figure 3: Forces and moments acting on an element $d s^{\prime}$.

where $m$ is the local moment, $\ell$ is the length of the rod, $\rho$ is the density, $\Omega$ is the rotational velocity, $y^{\prime}$ is the distance from the rotation axis, $s^{\prime}$ is the arc length and $\theta$ is the local angle of inclination.

For an elastica the local moment is related to the local curvature by

$$
m=E I \frac{d \theta}{d s^{\prime}}
$$

where $E I$ is the flexural rigidity of the rod. We introduce the nondimensional variables

$$
s=\frac{s^{\prime}}{\ell}, y=\frac{y^{\prime}}{\ell}, u=\int_{1}^{s} y d s, \quad J=\ell \quad \sqrt[4]{\frac{\rho \Omega^{2}}{E I}} .
$$

Using (1.2) and (1.3) the equation of equilibrium (1.1) becomes

$$
\left\{\begin{array}{l}
\frac{d^{2} \theta}{d s^{2}}=J^{4} u \cos \theta \\
\frac{d^{2} u}{d s^{2}}=\sin \theta
\end{array}\right.
$$

which is a system of two second order nonlinear differential equations in the two variables $u$ and $\theta$. The boundary conditions are

$$
\left\{\begin{array}{l}
\theta(0)=\alpha, \frac{d u}{d s}(0)=0, \\
\frac{d \theta}{d s}(1)=u(1)=0,
\end{array}\right.
$$

Upon using the transformations

$$
\left\{\begin{array}{l}
\theta=\varphi+\alpha, \\
\lambda=J^{2}, \\
v=\lambda u,
\end{array}\right.
$$

the equilibrium equations and the boundary conditions (1.4), (1.5) take the form

$$
\left\{\begin{array}{l}
\frac{d^{2} \varphi}{d s^{2}}=\lambda v \cos (\varphi+\alpha) \\
\frac{d^{2} v}{d s^{2}}=\lambda \sin (\varphi+\alpha) \\
\varphi(0)=\frac{d v}{d s}(0)=\frac{d \varphi}{d s}(1)=v(1)=0
\end{array}\right.
$$


Finally (1.7) can be written in the (integral) form

$$
\varphi=K(\varphi)
$$

where

$$
\begin{gathered}
K(\varphi)=\lambda^{2} \int_{0}^{1} \int_{0}^{1} G_{1}(s, \xi) G_{2}(\xi, \eta) \sin (\varphi(\eta)+\alpha) \cos (\varphi(\xi)+\alpha) d \eta d \xi, \\
G_{1}(s, \xi)= \begin{cases}s, & 0<s<\xi, \\
\xi, & \xi<s<1,\end{cases} \\
G_{1}(x, \xi)= \begin{cases}1-\xi, & 0<s<\xi, \\
1-s, & \xi<s<1\end{cases}
\end{gathered}
$$

The rest of this paper is organized in three sections. In the second section some preliminaries are given which will be used in the later sections. The third section is concerned with proofs of the existence and uniqueness of the solutions of (1.7). In the fourth section a perturbation solution of (1.7) is presented and compared with the numerical solution obtained in [3].

\section{PRELIMINARIES.}

Consider the linear eigenvalue problem

$$
\left\{\begin{array}{l}
\frac{d^{2} \varphi}{d s^{2}}=\lambda v \\
\frac{d^{2} v}{d s^{2}}=\lambda \varphi \\
\varphi(0)=\frac{d v}{d s}(0)=\frac{d \varphi}{d s}(1)=v(1)=0
\end{array}\right.
$$

It is well known [2] that the eigenvalues $\lambda_{n}$ of (2.1) are the squares of the roots of the equation

$$
\cos x \cosh x+1=0,
$$

corresponding to the "normalized" eigenfunctions

$$
\left\{\begin{array}{l}
V_{n}=\frac{1}{2} \lambda_{n}^{-3 / 2}\left(\sinh \sqrt{\lambda_{n}} s-\sin \sqrt{\lambda_{n}} s\right)+\frac{1}{2} \eta_{n}\left(\cosh \sqrt{\lambda_{n}} s+\cos \sqrt{\lambda_{n}} s\right) \\
\varphi_{n}=\lambda_{n}^{-1} \frac{d^{2} v_{n}}{d s^{2}} \\
\eta_{n}=-\lambda_{n}^{-3 / 2} \frac{\cosh \sqrt{\lambda_{n}}+\cos \sqrt{\lambda_{n}}}{\sinh \sqrt{\lambda_{n}}+\sin \sqrt{\lambda_{n}}}
\end{array}\right.
$$

It is also known [2] that (2.1) can be written in the integral form

$$
\varphi=A(\varphi)
$$

where

$$
A(\varphi)(s)=\lambda^{2} \int_{0}^{1} \int_{0}^{1} G_{1}(s, \xi) G_{2}(\xi, \eta) \varphi(\eta) d \eta d \xi
$$

and $G_{1}, G_{2}$ are the positive symmetric Green's functions defined, respectively, by (1.10) and (1.11), and that $A$ as an operator on $L_{2}(0,1)$ satisfies

$$
\|A\|=\frac{1}{\lambda_{0}^{2}}
$$

where $\lambda_{0}$ is the smallest eigenvalue of the linear problem (2.1). 


\section{EXISTENCE AND UNIQUENESS OF SOLUTIONS.}

In this section we show that the integral equation (1.8) has at least one solution for each $0<\alpha \leq \frac{\pi}{2}$ and each $\lambda$ and that for $\lambda<\lambda_{0}$ this solution is unique for all $0<\alpha \leq \frac{\pi}{2}$. The proof of the existence of solution is based upon a corollary of the Schauder fixed point theorem which we state here for completeness.

LEMMA 3.1 Let $B$ be a real Banach space, and let $K: B \rightarrow B$ be compact. Suppose that there is a priori bound $m>0$ such that every solution of $\varphi-t k \varphi=0,0 \leq t \leq 1$, satisfies $\|\varphi\| \leq m$. Then $K$ has a fixed point $\varphi$ such that $\|\varphi\| \leq m$.

The proof of existence of solution of (1.8) for each $0<\alpha \leq \frac{\pi}{2}$ and each $\lambda$ consists of verifying the conditions of Lemma 3.1. The compactness of the operator $K$ defined by (1.9) is proved in the following lemma.

LEMMA 3.2. For each $0<\alpha \leq \frac{\pi}{2}$ and each $\lambda$ the operator $K$ defined by (1.9) is compact operator on $L_{2}(0,1)$.

PROOF. Let $0<\alpha \leq \frac{\pi}{2}$ and $\lambda$ be fixed. The boundedness of the operator $A$ defined by (2.5), $\mid \sin \left(\varphi(s)+\alpha \mid \leq 1\right.$ and $|\cos (\varphi(s)+\alpha)| \leq 1$ imply that $K$ as an operator from $L_{2}(0,1)$ into $C([0,1])$ is bounded in the sense that it carries bounded subsets of $L_{2}(0,1)$ into bounded subsets in $C([0,1])$. Since the identity operator $i: C([0,1]) \rightarrow L_{2}(0,1)$ is compact it follows that $K$ is a compact operator on $L_{2}(0,1)$.

The uniqueness of the solution of (1.8) for $\lambda<\lambda_{0}$ follows from (2.6) and the observation that Frechet derivative of the operator $K$ is given by

$$
K^{\prime}(\varphi)=\lambda^{2} \int_{0}^{1} \int_{0}^{1} G_{1}(s, \xi) G_{2}(\xi, \eta) \operatorname{Cos}(\varphi(\eta)+\varphi(\xi)+2 \alpha) d \eta d \xi .
$$

We gather the results of this section in the following theorem.

THEOREM 3.3. Equation (1.8) has at least one solution for each $0<\alpha \leq \frac{\pi}{2}$ and each $\lambda$. Furthermore, for each $0<\alpha \leq \frac{\pi}{2}$ this solution is unique for $\lambda$ less than the smallest eigenvalue $\lambda_{0}$ of the linear problem (2.1).

\section{PERTURBATION SOLUTION FOR SMALL $\alpha>0$.}

In this section we present asymptotic expansions of the solution of (1.7) for small $\alpha>0$ in two cases. In the first case we take $\lambda<\lambda_{0}$. In this case the solution is unique and, as we will show below, it becomes invalid as $\lambda$ approaches $\lambda_{0}$. In the second case we take $\lambda>\lambda_{0}$ and assume that $\lambda-\lambda_{0}$ is small. In this case, we give an approximation of a critical $\tilde{\lambda}(\alpha)>\lambda_{0}$ such that for $\lambda>\tilde{\lambda}(\alpha)$ the uniqueness of the solution of (1.7) is lost. Finally, we compare our analytical approximation of $\tilde{\lambda}(\alpha)$ to its numerical approximation obtained in [3].

CASE 1: $\lambda<\lambda_{0}$.

For small $\alpha>0$ we linearize (1.7) about the "trivial" solution $\varphi \equiv 0, v \equiv 0$. To this end we write

$$
\begin{gathered}
\varphi(s)=\alpha \varphi_{1}(s)+O\left(\alpha^{2}\right) \\
v(s)=\alpha v_{1}(s)+O\left(\alpha^{2}\right)
\end{gathered}
$$

Substituting (4.1) into (1.7) and collecting terms that are linear in $\alpha$ yields

For $\lambda<\lambda_{0},(4.2)$ has a unique solution given by $\left\{\begin{array}{l}\frac{d^{2} \varphi_{1}}{d s^{2}}=\lambda v_{1}, \\ \frac{d^{2} v_{1}}{d s^{2}}=\lambda\left(\varphi_{1}+1\right), \\ \varphi_{1}(0)=\frac{d v_{1}}{d s}(0)=\frac{d \varphi_{1}}{d s}(1)=v_{1}(1)=0 .\end{array}\right.$ 
when $\lambda=0$ and

$$
\left\{\begin{array}{l}
n_{1} \equiv 0 \\
\varphi_{1} \equiv 0
\end{array}\right.
$$

$$
\left\{\begin{array}{l}
r_{1}=A \cos \sqrt{\lambda} s+B \sin \sqrt{\lambda} s+C e^{\sqrt{\lambda} s}+D e^{-\sqrt{\lambda} s}, \\
\varphi_{1}=\frac{1}{\lambda} r_{1}^{\prime \prime}-1
\end{array}\right.
$$

when $\lambda \neq 0$, where $A, B, C$ and $D$ are the constants determined by

$$
\left\{\begin{array}{l}
A=\frac{-\cos \sqrt{\lambda} \cosh \sqrt{\lambda}-\sin \sqrt{\lambda} \sinh \sqrt{\lambda}-1}{2(\cos \sqrt{\lambda} \cosh \sqrt{\lambda}+1)} \\
B=\frac{-\cos \sqrt{\lambda} \sinh \sqrt{\lambda}-2 \cosh \sqrt{\lambda} \sinh \sqrt{\lambda}-\sin \sqrt{\lambda} \cosh \sqrt{\lambda}}{2(\cos \sqrt{\lambda} \cosh \sqrt{\lambda}+1)} \\
C=\frac{1+A-B}{2} \\
D=\frac{1+A+B}{2}
\end{array}\right.
$$

From (4.5) we see that the solutions (4.4) become unbounded as $\lambda \rightarrow \lambda_{0}$ which render the expansions (4.1) invalid. This latter case is treated differently below.

CASE 2: $\lambda>\lambda_{0}$ and $\lambda-\lambda_{0}$ is small.

As mentioned above, as $\lambda \rightarrow \lambda_{0}$, the expansions (4.1) are not valid. For $\lambda=\lambda_{0}$ the linear problem (2.1) has the unique (up to multiplicative constant) solution (2.3) with $\lambda_{n}=\lambda_{0}$. For $\alpha=0, \lambda=\lambda_{0}$ is a bifurcation point where a new branch of solutions emerges. For $\alpha \neq 0$ this bifurcation is perturbed. Our goal here is to analyze this perturbed bifurcation situation.

The present problem now involves two small parameters $\alpha$ and $\lambda-\lambda_{0}$. We introduce a small "amplitude" parameter $\varepsilon$ and seek solutions of (1.7) in the form

$$
\left\{\begin{array}{l}
\varphi=\varepsilon \varphi_{1}+\varepsilon^{2} \varphi_{2}+\varepsilon^{3} \varphi_{3}+O\left(\varepsilon^{4}\right), \\
v=\varepsilon v_{1}+\varepsilon^{2} v_{2}+\varepsilon^{3} v_{3}+O\left(\varepsilon^{4}\right),
\end{array}\right.
$$

such that $\left(\varphi_{i}, v_{i}\right), i>1$, are orthogonal to $\left(\varphi_{1}, v_{1}\right)$. We also expand $\alpha$ and $\lambda-\lambda_{0}$ in powers of $\varepsilon$. It turns out that, for bounded solutions to exist, these expansions must be of the following form

$$
\left\{\begin{array}{l}
\alpha=\varepsilon^{3}+O\left(\varepsilon^{4}\right) \\
\lambda=\lambda_{0}+\varepsilon^{2} \lambda_{1}+\varepsilon^{3} \lambda_{2}+O\left(\varepsilon^{4}\right) .
\end{array}\right.
$$

Substituting (4.6) and (4.7) into (1.7) and collecting like powers of $\varepsilon$ yields to the linear boundary value problem (2.1) with $\lambda=\lambda_{0}$. Thus we can write $v_{1}$ and $\varphi_{1}$ as

$$
\begin{aligned}
& \varphi_{1}=A \varphi_{0}, \\
& v_{1}=A v_{0},
\end{aligned}
$$

where $\varphi_{0}$ and $v_{0}$ are those functions defined by (2.3) with $\lambda_{n}=\lambda_{0}$ and $A$ is an undetermined constant. 
The system for the second order terms is also the same as (2.1) with $\lambda=\lambda_{0}$. In order to make the solutions of this systems unique we require that they are orthogonal to their respective first order quantities. This leads to

$$
\begin{aligned}
& \varphi_{2} \equiv 0, \\
& v_{2} \equiv 0 .
\end{aligned}
$$

This leaves $A$ and $\lambda_{1}$ undetermined.

The system for the third order quantities is

$$
\left\{\begin{array}{l}
\frac{d^{2} \varphi_{3}}{d s^{2}}=\lambda_{o} v_{3}+\lambda_{1} v_{1}-\frac{1}{2} \lambda_{0} \varphi_{1}^{2}, \\
\frac{d^{2} v_{3}}{d s^{2}}=\lambda_{0} \varphi_{3}+\lambda_{0}-\frac{1}{6} \lambda_{0} \varphi_{1}^{3}+\lambda_{1} \varphi_{1}, \\
\varphi_{3}(0)=\frac{d v_{3}}{d s}(0)=\frac{d \varphi_{3}}{d s}(1)=v_{3}(1)=0 .
\end{array}\right.
$$

The solvability condition for $\left(\varphi_{3}, v_{3}\right)$ requires the right hand side of $(4.10)$ to be orthogonal to $\left(\varphi_{0}, v_{0}\right)$. After some simplification this leads to the equation

$$
\left\{\begin{array}{c}
A^{3}-\gamma \lambda_{1} A-\delta=0, \\
\gamma=\frac{\int_{0}^{1}\left(\varphi_{0}^{\prime}\right)^{2} d s}{\lambda_{0} \int_{0}^{1}\left(\varphi_{0}^{\prime} \varphi_{0}\right)^{2} d s}, \\
\delta=\frac{1}{2 \lambda_{0} \int_{0}^{1}\left(\varphi_{0}^{\prime} \varphi_{0}\right)^{2} d s,}
\end{array}\right.
$$

The first equation of (4.7) implies that $\varepsilon \approx \alpha^{1 / 3}$ and hence in this "singular" case the solution $(\varphi, v)$ depends on $\alpha$ through powers of $\alpha^{1 / 3}$. Equation (4.11) reveals the following information about the bifurcation picture. For $\lambda_{1}<\lambda_{1}^{*} \equiv \frac{3}{\gamma}\left(\frac{\delta}{2}\right)^{2 / 3}$ equation (4.11) has a unique real solution for $A$ and for $\lambda_{1}>\lambda_{1}^{*}$ it has three real solutions. This enables us to approximate the critical value of $\lambda$, beyond which the uniqueness of the solution of (1.7) is lost, by

$$
\tilde{\lambda}=\lambda_{0}+\frac{3}{\gamma}\left(\frac{\delta}{2}\right)^{2 / 3} \alpha^{2 / 3}
$$

for small $\alpha>0$.

Finally, we compare our analytical results obtained from equation (4.12) to the (exact) numerical approximations given by Figure 9 of [3]. Evaluating the parameters $\delta, \gamma$ defined by (4.11) and substituting into (4.12) we obtain the following approximation of the critical value of $\lambda$ beyond which multiple solutions of (1.7) exist

$$
\tilde{\lambda}=3.516015+3.385993 \alpha^{2 / 3} \text {, }
$$

for small $\alpha>0$. It follows from (1.6) that the critical values $J_{M i n}$ defined in [3] can be approximated by

$$
J_{M i n} \approx \tilde{J} \equiv \tilde{\lambda}^{1 / 2}
$$


for small $\alpha>0$. In Figure 4 below we compare the analytical approximations of $J_{M}$ obtained from equation (4.14) to their numerical values given in [3] for all $0 \leq \alpha \leq \frac{\pi}{2}$. We notice that the difference $J_{M i n}-\tilde{J} \geq 0$, is very small for small values of $\alpha$ and increases as $\alpha$ increases.

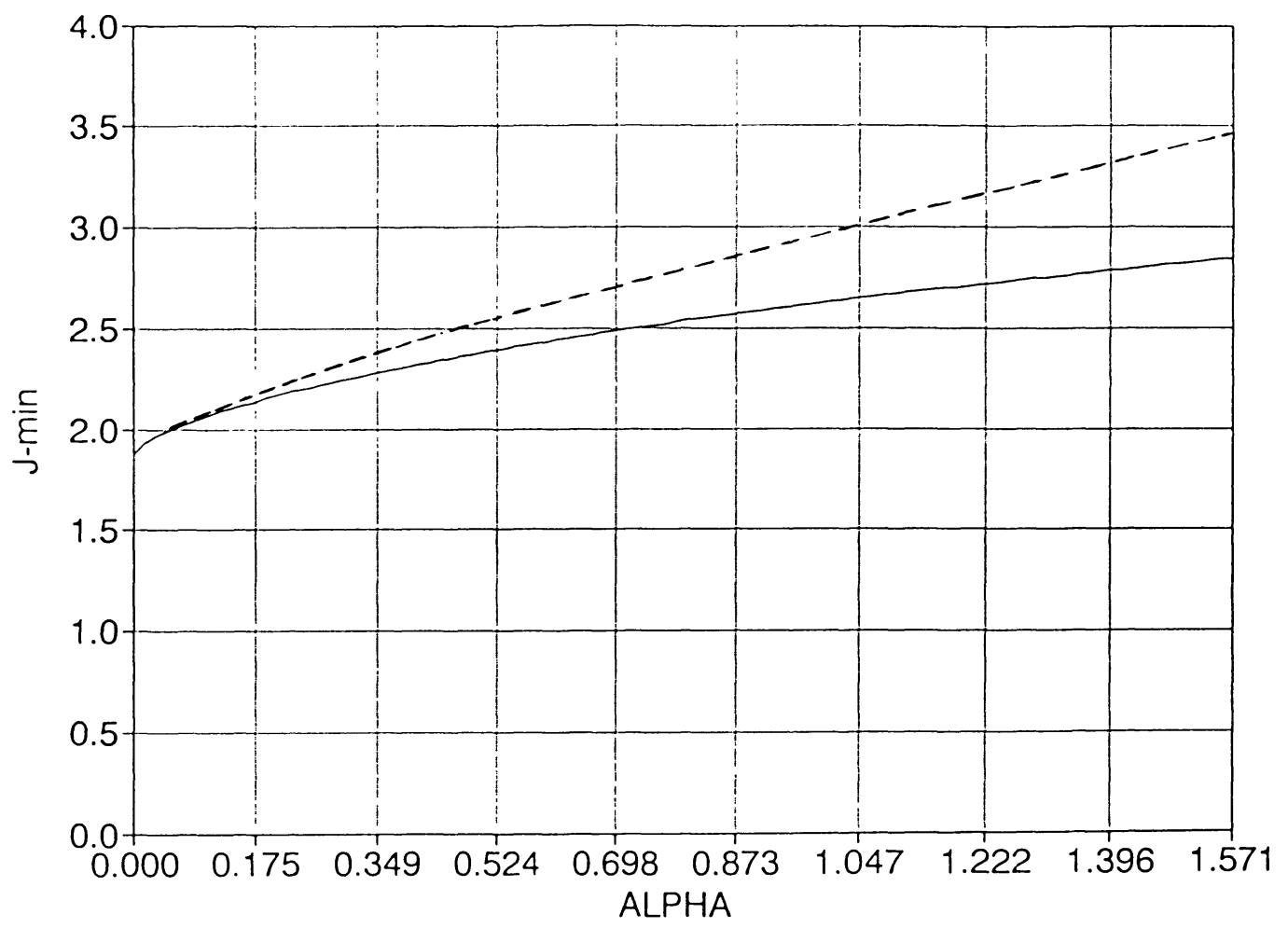

Figure 4: Critical values $J_{M \text { in }}$ beyond which multiple solutions exist. Dashed curve is exact.

ACKNOWLEDGEMENT. I would like to thank Professor C.Y. Wang, Department of Mathematics, Michigan State University, for suggesting the problem of this paper and for his helpful discussions and comments, Professor D. Lund, Department of Mathematics, University of Wisconsin-Eau Claire, for his help with the graphs of Figure 4 and Mrs. Sue Johnson for typing the manuscript of this paper.

\section{REFERENCES}

1. ODEH, F. \& TADJBAKHSH, I., A nonlinear eigenvalue problem for rotating rods, Arch. Ration. Mech. Anal. 20 (1965), 81-94.

2. WANG, C.Y., On the bifurcation solutions of an axially rotating rod, Q.J. Mech. Appl. Math. $\underline{35}$ (3) (1982), 391-402.

3. WANG, C.Y., Nonlinear deformation of a slender rod rotated at one end, ZMAP 41 (1990), 900-912. 


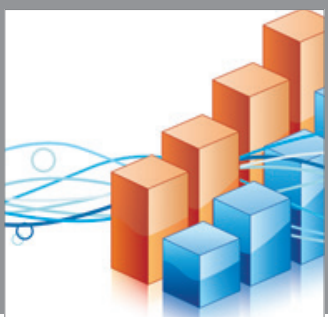

Advances in

Operations Research

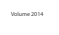

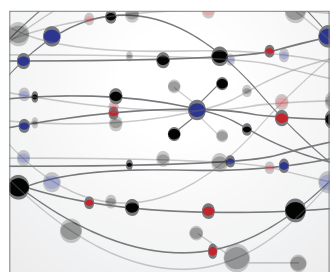

\section{The Scientific} World Journal
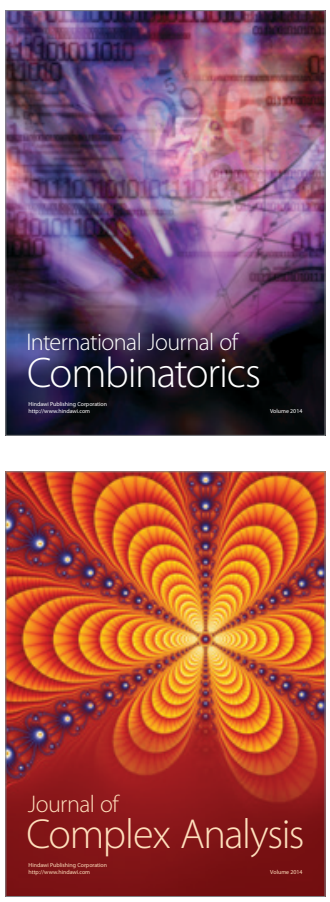

International Journal of

Mathematics and

Mathematical

Sciences
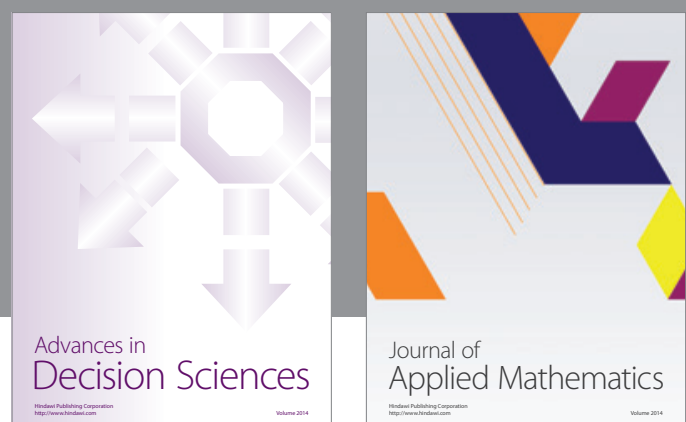

Journal of

Applied Mathematics
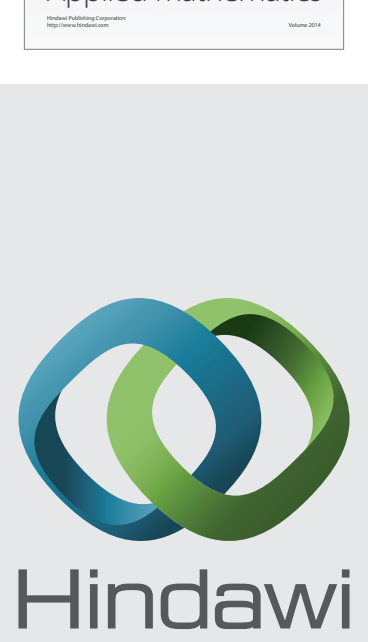

Submit your manuscripts at http://www.hindawi.com
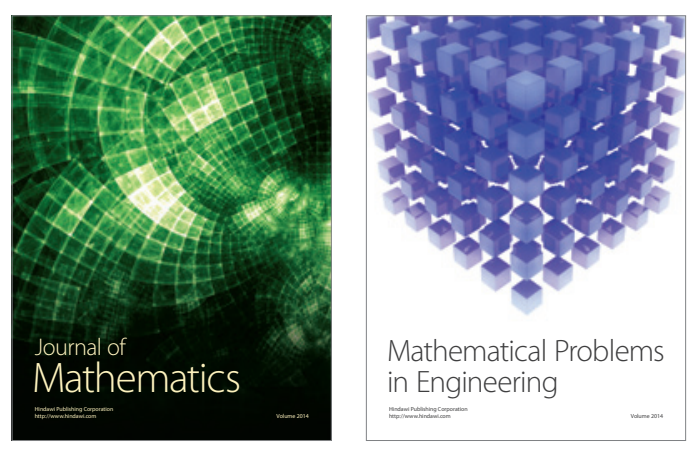

Mathematical Problems in Engineering
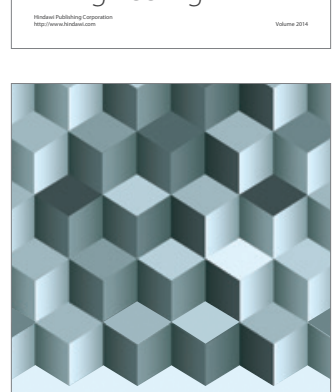

Journal of

Function Spaces
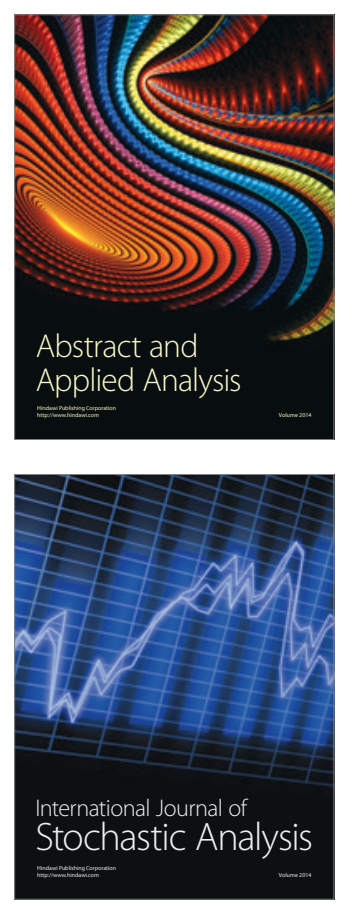

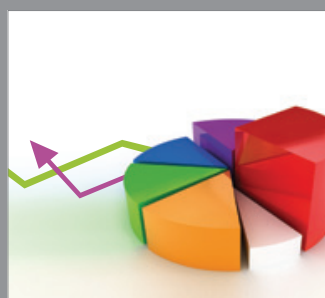

ournal of

Probability and Statistics

Promensencen
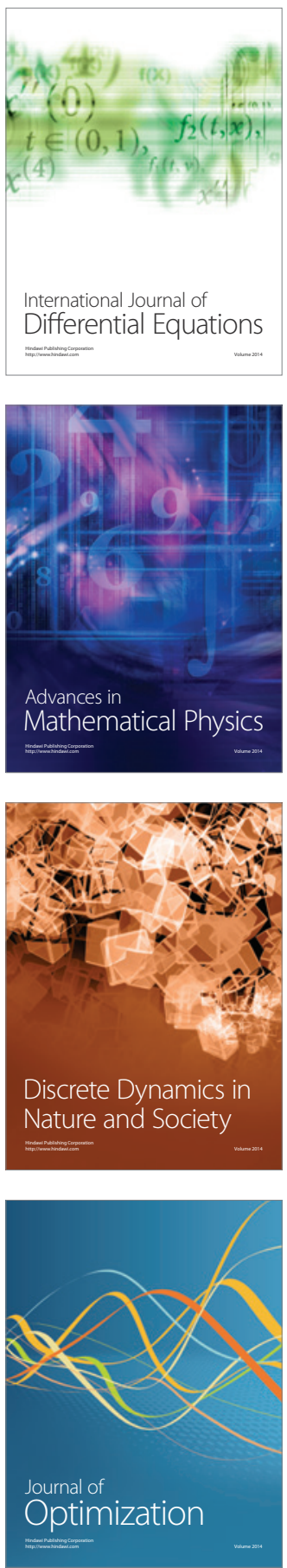